\title{
A Hybrid Algorithm for the Permutation Flow Shop Scheduling Problem
}

\author{
Arindam Chakravorty ${ }^{1}$ and Dipak Laha ${ }^{2}$ \\ 1 Department of Information Technology, St. Thomas' College of Engineering \\ and Technology, Kolkata 700023, India \\ 2 Department of Mechanical Engineering, Jadavpur University, Kolkata 700032, India
}

\begin{abstract}
This paper considers the application of IA for the classic permutation flow shop scheduling problem. We present a hybrid version of constructively built IA combining with the SA for the n-job, m-machine permutation flow shop scheduling problem to minimize makespan. Based on all the Taillard's benchmark problems, the computational results suggest that the proposed method is very competitive with the existing methods in the literature.
\end{abstract}

Keywords: Scheduling, Immune algorithm, Simulated annealing, Permutation flow shop, Makespan, Shift neighborhood search.

\section{Introduction}

In recent times, with the emergence of a variety of evolutionary algorithms, such as GA, ACO, PSO, and DE, another important technique is the immune algorithm (IA), which is guided by the principles concerning the concepts and functions extracted from theoretical immunology. Dasgupta [1, and Hart and Timmis [2] have given an in depth overview of this algorithm. This paper suggests an improved IA by incorporating construction based heuristic, neighborhood solutions and suitable operators of the algorithm. To improve the IA, we hybridize it with SA. Since the flow shop scheduling problem is known to be NP-hard [3], approximate algorithms including computational intelligence based algorithms provide near-optimal/optimal solutions, especially on more difficult, complex search spaces.

Noteworthy research on application of IA to flow shop scheduling has been carried out by Zandieh et al. [4], Kai and Gen-ke, [5], Kurz and Askin [6], Bagheri et al. [7], and Engin and Dyen [8. Applications of ACO to flow shop scheduling have been studied by Rajendran and Ziegler [9], MMAS [10], Yang and Liao [11], Ahmadizar and Barzinpour [12, and Ahmadizar [13. In this paper, we propose a hybrid IA combining with SA for the permutation flow shop scheduling problem to minimize the makespan.

\section{Problem Definition}

We denote $p_{i j}$ as the processing time for each job $i, i \varepsilon n$ on each machine $j, j \varepsilon m$. The objective is to minimize the makespan for permutation flow shop scheduling. 
Let $\Pi$ denote all permutations of $n$-job schedules in the solution search space for the flow shop problem. Let $\pi$ represent a job permutation of schedule, $\pi \varepsilon \Pi$ and $\pi=\left\{\pi_{1}, \pi_{2}, \ldots, \pi_{n},\right\}$. Then, the completion time of $\pi_{j}$ on machine $m, C\left(\pi_{j}, m\right)$ is given as: $C\left(\pi_{j}, m\right)=\max \left\{C\left(\pi_{j-1}, k\right), C\left(\pi_{j}, k-1\right)+p_{\pi_{j}, k}\right\} \ldots$ (1)

Then, the makespan of a schedule of $\mathrm{n}$-job is defined as: $M S(\pi)=C\left(\pi_{n}, m\right) \ldots(2)$

Therefore, for $n / m / p r m u / C_{\max }$ problem, the objective is to determine a permutation schedule $\pi^{*} \varepsilon \Pi$ such that: $M S\left(\pi^{*}\right)=\min _{\pi \varepsilon \Pi} M S(\pi) \ldots(3)$

\section{Proposed Algorithm}

The proposed algorithm has three major phases, namely, initial solution phase, construction phase and improvement phase. In the initial solution phase, an initial sequence of jobs is created. Construction phase is used to build the sequence of $n$ jobs incrementally by selecting jobs from the initial sequence using IA. Finally, in improvement phase, the solution is further improved by employing neighbourhood search and SA.

Phase 1: Initial sequence generation - The jobs are arranged in descending order of their total processing time $\left(\mathrm{TPT}=\sum_{j=1}^{m} p_{i j}\right)$ to create an initial (seed) sequence.

Phase 2: Construction procedure

Algorithm of Construction phase:

1. Generate an initial sequence of $n$ jobs using phase 1 . Call it seed sequence.

2. Select first $\mathrm{r}$ jobs and permute them in $\mathrm{r}$ ! sequences. Select best $\mathrm{M},(M \leq r !)$ sequences on the basis of makespan values from these sequences.

3 . Set $\mathrm{k}=\mathrm{r}+1$. Repeat steps 5 to 14 while $k \leq n$.

4. For each of the sequences selected in step 2 repeat step 6 .

5. Pick up the $k^{\text {th }}$ job from the seed sequence and place it in $\mathrm{k}$ positions of the sequence.

6. Sort the $\mathrm{k} \times \mathrm{M}$ sequences of $\mathrm{k}$ jobs each obtained in step 6 in ascending order of makespan and select the best $\mathrm{M}$ sequences of $\mathrm{k}$ jobs each.

7. Clone the M sequences based on Roulette wheel selection principle and create $\mathrm{M}$ clone sequences.

8. For each of the $\mathrm{M}$ clone sequences use partially mapped crossover with a randomly selected clone from the rest $(\mathrm{M}-1)$ clones. This creates a $2 \mathrm{M}$ strong clone pool.

9. For $\mathrm{p}=1$ to $\mathrm{p}=2 \mathrm{M}$ repeat steps 11 and 12 .

10. Pairwise mutate clone and call the new sequence as newsequence. If makespan of newsequence is less than makespan of clone then set clone $=$ newsequence.

11. Inverse mutate $p^{\text {th }}$ clone (clone) and call the new sequence as newsequence. If makespan of newsequence is less than makespan of clone then set clone $=$ newsequence. 
12. Select best $\mathrm{M}$ clones on the basis of makespan from the pool of $2 \mathrm{M}$ clones. Set $\mathrm{k}=\mathrm{k}+1$.

Parameters selection in construction phase: The value of $\mathrm{r}$ has been optimized to 4 . The algorithm has been tested with $\mathrm{M}=10,12,15,18,20$ and 24 and optimized to $\mathrm{M}=20$.

Phase 3: Improvement procedure

Algorithm of Improvement phase:

1. The best sequence obtained in the construction phase is selected as the best-so-far sequence (best) with makespan $=M S_{\text {best }}$.

2. Let s represent the current sequence, and $M S_{s}$ the corresponding makespan.

3. Set $s \leftarrow$ best and $M S_{s} \leftarrow M S_{\text {best }}$.

4. Initialize max-count and the start temperature $\left(T_{1}\right)$. Set count $=1$.

5. Apply the forward shift mechanism to s, separately n-1 times (each case being independent of the others), by randomly picking, in each case, a pair of jobs at two distinct positions $\mathrm{h}$ and $\mathrm{i}$ (where $1 \leq h<i \leq n$ ). This produces $\mathrm{n}-1$ neighboring sequences. Find the best of these sequences $s^{\prime}$, with a makespan of $M S_{S^{\prime}}$.

6. If $M S_{s^{\prime}}<M S_{s}$ then \{

6.1 Set the new sequence as the current sequence (that is, set $s \leftarrow s^{\prime}$ and $\left.M S_{s} \leftarrow M S_{s^{\prime}}\right)$

6.2 If $M S_{s^{\prime}}<M S_{\text {best }}$ then set best $\leftarrow s^{\prime}$ and $\left.M S_{\text {best }} \leftarrow M S_{s^{\prime}} ;\right\}$

7.Apply the backward shift mechanism to s, separately n-1 times (each case being independent of the others), by randomly picking, in each case, a pair of jobs at two distinct positions $\mathrm{h}$ and $\mathrm{i}$ (where $1 \leq h<i \leq n$ ). This produces $\mathrm{n}-1$ neighboring sequences. Find the best of these sequences $s^{\prime}$, with a makespan of $M S_{s^{\prime}}$.

8.If $M S_{s^{\prime}}<M S_{s}$ then \{

8.1 Set the new sequence as the current sequence (that is, set $s \leftarrow s^{\prime}$ and $\left.M S_{s} \leftarrow M S_{s^{\prime}}\right)$

8.2 If $M S_{s^{\prime}}<M S_{\text {best }}$ then set best $\leftarrow s^{\prime}$ and $\left.M S_{\text {best }} \leftarrow M S_{s^{\prime}} ;\right\}$

Else \{

8.3 With probability $\exp \left(\left(M S_{s}-M S_{s^{\prime}}\right) /\left(M S_{s} * T_{1}\right)\right)$ set $s \leftarrow s^{\prime}$ and $M S_{s} \leftarrow$ $\left.M S_{s^{\prime}}\right\}$

9. Set count $=$ count $+1, T_{1}=0.9 * T_{1}$. If count $\leq$ max-count go to step 5

Output: the best-so-far sequence (best) and its makespan, $M S_{\text {best }}$ as the final solution.

Parameters in improvement phase are taken as: max-count $=200,250,300$, $500,800,1000$ and optimized to max-count $=500 . \mathrm{T}_{1}=100,150,200,250,300$ and optimized to $\mathrm{T}_{1}=200$.

\section{Computational Results}

The proposed hybrid immune algorithm has been coded in Java and run on Intel Atom 1.6 GHz with $2 \mathrm{~GB}$ memory PC. To evaluate the solution quality and 
efficiency of the proposed method, the experiments are carried out based on Taillard's benchmark problems [14]. For each problem instance of a problem size, the integer processing time matrix is generated following a uniform discrete distribution $[1,99]$ for each known random number seed value given by Taillard. For each combination of job and machine, 10 independent problem instances are created, producing 120 problem instances in all. To assess the performance of proposed IA with other algorithms, average relative percentage deviation (AR) and the CPU time are used. AR is defined as: $A R=\frac{\sum_{i=1}^{N P}\left(M S_{i}-U B_{i}\right) / U B_{i}}{N P} \times 100 \ldots$ (4)

where, $M S_{i}$ and $U B_{i}$ respectively represent the makespan obtained using an algorithm and the corresponding upper bound for a particular seed value as given in Taillard [14. The upper bound (UB) is the best value close to the optimal makespan as good as possible and is given by Taillard. Due to the stochastic nature, the proposed algorithm is run for five trials instead of one trial for each problem instance of a problem size and the best and mean values are computed. Then, AR for a problem size is computed using Equation (4), considering each of best and mean makespan values with respect to the UB values.

The proposed algorithm is compared with M-MMAS 9], PACO [9], ACS [1], ACA [12], and NACA [13]. It may be noted that the above mentioned algorithms due to their probabilistic nature have been applied for each problem instance for five trials and the best and mean values have been chosen. Also, the corresponding average CPU time (in seconds) required for these algorithms has been reported. The detailed results are found in Ahmadizar [13. The above existing algorithms were run on a Pentium 4, 2GHz PC which is slightly faster or at least same as our computer.

The comparative $A R_{\text {best }}, A R_{\text {mean }}$, and CPU times of the proposed IA with and without SA and the five competing algorithms are reported in Tables 1 and 2. The computational results reveal that the IA with SA performs significantly

Table 1. Comparison with NACA, M-MMAS and PACO (keeping M-MMAS and PACO same CPU time as that of NACA)

\begin{tabular}{ccccccccccccccc}
\hline \multicolumn{10}{c}{ IA } & \multicolumn{1}{c}{ NACA } & M-MMAS & PACO \\
\hline \multicolumn{10}{c}{ Without SA } & \multicolumn{1}{c}{ With SA } \\
\hline$n$ & $m$ & $A R_{\text {best }}$ & $A R_{\text {mean }}$ & $A R_{\text {best }}$ & $A R_{\text {mean }}$ & Time $(s)$ & $A R_{\text {best }}$ & $A R_{\text {mean }}$ & Time $(s)$ & $A R_{\text {best }}$ & $A R_{\text {mean }}$ & $A R_{\text {best }} A R_{\text {mean }}$ \\
\hline 20 & 5 & 0.669 & 1.339 & 0.172 & 0.593 & 0.43 & 0.000 & 0.130 & 0.84 & 0.127 & 0.306 & 0.000 & 0.138 \\
& 10 & 1.817 & 2.716 & 0.486 & 0.958 & 0.45 & 0.079 & 0.392 & 1.57 & 0.447 & 0.747 & 0.181 & 0.458 \\
& 20 & 1.606 & 2.472 & 0.536 & 0.867 & 0.56 & 0.102 & 0.321 & 3.61 & 0.315 & 0.552 & 0.111 & 0.280 \\
50 & 5 & 0.212 & 0.448 & 0.072 & 0.209 & 1.47 & 0.011 & 0.070 & 5.03 & 0.057 & 0.093 & 0.053 & 0.076 \\
& 10 & 1.294 & 2.364 & 0.403 & 1.176 & 2.19 & 0.257 & 0.742 & 11.14 & 0.843 & 1.232 & 0.761 & 1.186 \\
& 20 & 2.916 & 4.054 & 1.333 & 1.925 & 3.51 & 1.252 & 1.808 & 22.71 & 1.561 & 2.040 & 1.781 & 2.165 \\
100 & 5 & 0.127 & 0.262 & 0.010 & 0.081 & 8.40 & -0.006 & 0.076 & 19.46 & 0.036 & 0.094 & 0.030 & 0.073 \\
& 10 & 0.933 & 1.294 & 0.404 & 0.660 & 12.11 & 0.282 & 0.557 & 43.68 & 0.463 & 0.583 & 0.428 & 0.585 \\
& 20 & 2.322 & 2.682 & 0.714 & 1.143 & 16.08 & 0.761 & 1.287 & 93.94 & 1.183 & 1.489 & 1.274 & 1.565 \\
200 & 10 & 0.204 & 0.464 & -0.179 & 0.012 & 77.34 & 0.150 & 0.341 & 177.39 & 0.289 & 0.469 & 0.275 & 0.428 \\
& 20 & 0.706 & 1.052 & -0.043 & 0.181 & 105.38 & 0.306 & 0.744 & 389.67 & 0.571 & 0.809 & 0.708 & 0.957 \\
500 & 20 & -0.376 & -0.376 & -0.794 & -0.794 & 1440 & 0.230 & 0.515 & 2474.4 & 0.408 & 0.526 & 0.490 & 0.615 \\
\hline Average: & 1.036 & 1.564 & 0.260 & 0.584 & 138.99 & 0.285 & 0.582 & 270.29 & 0.525 & 0.745 & 0.508 & 0.711 \\
\hline
\end{tabular}


Table 2. Comparison with ACS and ACA

\begin{tabular}{|c|c|c|c|c|c|c|c|c|}
\hline & & \multicolumn{3}{|c|}{ IA } & \multicolumn{2}{|c|}{ ACS } & \multicolumn{2}{|c|}{$\mathrm{ACA}$} \\
\hline & \multicolumn{2}{|c|}{ Without SA } & \multicolumn{2}{|c|}{ With SA } & & & & \\
\hline$n$ & $m$ & $A R_{\text {best }}$ & $A R_{\text {best }}$ & Time $(s)$ & $A R_{\text {best }}$ & Time $(s)$ & $A R_{\text {best }}$ & Time $(s)$ \\
\hline \multirow[t]{3}{*}{20} & 5 & 0.669 & 0.172 & 0.43 & 1.19 & 3.67 & 0.368 & 0.44 \\
\hline & 10 & 1.817 & 0.486 & 0.45 & 1.70 & 4.00 & 0.831 & 0.50 \\
\hline & 20 & 1.606 & 0.536 & 0.56 & 1.60 & 5.33 & 0.944 & 0.63 \\
\hline \multirow[t]{3}{*}{50} & 5 & 0.212 & 0.072 & 1.47 & 0.43 & 14.67 & 0.085 & 2.77 \\
\hline & 10 & 1.294 & 0.403 & 2.19 & 1.89 & 18.00 & 1.241 & 3.73 \\
\hline & 20 & 2.916 & 1.333 & 3.51 & 2.71 & 24.33 & 1.990 & 5.91 \\
\hline \multirow[t]{3}{*}{100} & 5 & 0.127 & 0.010 & 8.40 & 0.22 & 54.33 & 0.070 & 14.15 \\
\hline & 10 & 0.933 & 0.404 & 12.11 & 1.22 & 65.67 & 1.059 & 21.93 \\
\hline & 20 & 2.322 & 0.714 & 16.08 & 2.22 & 88.00 & 1.833 & 37.79 \\
\hline \multirow[t]{2}{*}{200} & 10 & 0.204 & -0.179 & 77.34 & 0.64 & 275.33 & 0.434 & 141.52 \\
\hline & 20 & 0.706 & -0.043 & 105.38 & 1.30 & 631.67 & 1.236 & 254.06 \\
\hline 500 & 20 & -0.376 & -0.794 & 1440 & 1.68 & 5133.00 & 1.444 & 3744.3 \\
\hline \multicolumn{2}{|c|}{ Average: } & 1.036 & 0.260 & 138.99 & 1.40 & 526.50 & 0.961 & 352.31 \\
\hline
\end{tabular}

better than all the algorithms except NACA where it shows comparable solution. However, the IA without SA produces inferior solutions compared to other methods.

\section{Conclusion}

In this paper, we present a hybrid version of the immune algorithm and the simulated annealing to enhance the quality of solution while retaining the same order of computational time. The proposed algorithm employs hybridization of two evolutionary computational methods which has enabled better searching options. This approach has shown to yield results that are better or competitive than the other notable evolutionary algorithms.

\section{References}

[1] Dasgupta, D.: Artificial immune systems and their applications. Springer (1999)

[2] Hart, E., Timmis, J.: Applications areas of AIS: The past, the present and the future. Applied Soft Computing 8, 191-201 (2008)

[3] Gonzalez, T., Sahani, S.: Flow shop and job shop scheduling: complexity and approximation. Operations Research 26, 36-52 (1978)

[4] Zandieh, M., Fatemi Fhomi, S.M.T., Moattar Husseini, S.M.: An immune algorithm approach to hybrid flow shops scheduling with sequence-dependent setup times. Applied Mathematics and Computation 180, 111-127 (2006)

[5] Kai, S., Gen-ke, Y.: Hybrid artificial immune system and extremal optimization algorithm for permutation flowshop scheduling problem. Journal of Shanghai Univ (Engl Ed) 12(4), 352-357 (2008) 
[6] Kurz, M.E., Askin, R.G.: Scheduling flexible flow lines with sequence-dependent setup times. European Journal of Operational Research 159, 66-82 (2004)

[7] Bagheri, A., Zandieh, M., Mahdavi, I., Yazdani, M.: An artificial immune algorithm for the flexible job-shop scheduling problem. Future Generation Computer Systems 26, 533-541 (2010)

[8] Engin, O., Doyen, A.: A new approach to solve hybrid flow shop scheduling problems by artificial immune system. Future Generation Computer Systems 20, 1083-1095 (2004)

[9] Rajendran, C., Ziegler, H.: Ant-colony algorithms for permutation flowshop scheduling to minimize makespan/total flowtime of jobs. European Journal of Operational Research 155, 426-438 (2004)

[10] Stuetzle, T.: An ant approach for the flow shop problem. Proceedings of the 6th European Congress on Intelligent Techniques and Soft Computing (EUFIT) 3, 1560-1564 (1998)

[11] Ying, K.C., Liao, C.J.: An ant colony system for permutation flow-shop sequencing. Computers \& Operations Research 31, 791-801 (2004)

[12] Ahmadizar, F., Barzinpour, F.: A hybrid algorithm to minimize makespan for the permutation flow shop scheduling problem. International Journal of Computational Intelligence Systems 3, 853-861 (2010)

[13] Ahmadizar, F.: A new ant colony algorithm for makespan minimization in permutation flow shops. Computers \& Industrial Engineering 63, 355-361 (2012)

[14] Taillard, E.: Benchmarks for basic scheduling problems. European Journal of Operational Research 64, 278-285 (1993) 